\title{
Identifying obstructive sleep apnoea patients responsive to supplemental oxygen therapy
}

\author{
Scott A. Sands ${ }^{1,2}$, Bradley A. Edwards ${ }^{1,3,4,5}$, Philip I. Terrill ${ }^{6}$, James P. Butler ${ }^{1}$, \\ Robert L. Owens ${ }^{1,7}$, Luigi Taranto-Montemurro ${ }^{1}$, Ali Azarbarzin ${ }^{1}$, \\ Melania Marques ${ }^{1}$, Lauren B. Hess ${ }^{1}$, Erik T. Smales ${ }^{1}$, Camila M. de Melo', \\ David P. White ${ }^{1}$, Atul Malhotra ${ }^{1,7}$ and Andrew Wellman ${ }^{1}$
}

Affiliations: 'Division of Sleep and Circadian Disorders, Brigham and Women's Hospital and Harvard Medical School, Boston, MA, USA. ${ }^{2}$ Dept of Allergy, Immunology and Respiratory Medicine and Central Clinical School, The Alfred and Monash University, Melbourne, Australia. ${ }^{3}$ Sleep and Circadian Medicine Laboratory, Dept of Physiology, Monash University, Melbourne, Australia. ${ }^{4}$ School of Psychological Sciences, Monash University, Melbourne, Australia. ${ }^{5}$ Monash Institute of Cognitive and Clinical Neurosciences, Monash University, Melbourne, Australia. ${ }^{6}$ School of Information Technology and Electrical Engineering. The University of Queensland, Brisbane, Australia. ${ }^{7}$ Division of Pulmonary, Critical Care and Sleep Medicine, University of California San Diego, La Jolla, CA, USA.

Correspondence: Scott A. Sands, Division of Sleep Medicine, Brigham and Women's Hospital, 221 Longwood Avenue, Boston 02115, MA, USA. E-mail: sasandsabwh.harvard.edu

@ERSpublications

A subgroup of patients with obstructive sleep apnoea who benefit from stabilising ventilatory control with supplemental oxygen therapy can be recognised by estimating pathophysiological mechanisms from a routine diagnostic sleep study http://ow.ly/yeVp30lewG4

Cite this article as: Sands SA, Edwards BA, Terrill PI, et al. Identifying obstructive sleep apnoea patients responsive to supplemental oxygen therapy. Eur Respir J 2018; 52: 1800674 [https://doi.org/10.1183/ 13993003.00674-2018].

ABSTRACT A possible precision-medicine approach to treating obstructive sleep apnoea (OSA) involves targeting ventilatory instability (elevated loop gain) using supplemental inspired oxygen in selected patients. Here we test whether elevated loop gain and three key endophenotypic traits (collapsibility, compensation and arousability), quantified using clinical polysomnography, can predict the effect of supplemental oxygen on OSA severity.

36 patients (apnoea-hypopnoea index (AHI) >20 events. $\mathrm{h}^{-1}$ ) completed two overnight polysomnographic studies (single-blinded randomised-controlled crossover) on supplemental oxygen (40\% inspired) versus sham (air). OSA traits were quantified from the air-night polysomnography. Responders were defined by $\mathrm{a} \geqslant 50 \%$ reduction in AHI (supine non-rapid eye movement). Secondary outcomes included blood pressure and self-reported sleep quality.

Nine of 36 patients $(25 \%)$ responded to supplemental oxygen $(\Delta \mathrm{AHI}=72 \pm 5 \%)$. Elevated loop gain was not a significant univariate predictor of responder/non-responder status (primary analysis). In post hoc analysis, a logistic regression model based on elevated loop gain and other traits (better collapsibility and compensation; cross-validated) had $83 \%$ accuracy ( $89 \%$ before cross-validation); predicted responders exhibited an improvement in OSA severity $(\triangle \mathrm{AHI} 59 \pm 6 \%$ versus $12 \pm 7 \%$ in predicted non-responders, $\mathrm{p}=0.0001)$ plus lowered morning blood pressure and "better" self-reported sleep.

Patients whose OSA responds to supplemental oxygen can be identified by measuring their endophenotypic traits using diagnostic polysomnography.

This article has supplementary material available from erj.ersjournals.com

This study is registered at ClinicalTrials.gov with identifier number NCT01751971. Data will be made available to qualified scientists upon request.

Received: April 092018 | Accepted after revision: July 292018

Copyright OERS 2018 


\section{Introduction}

Around half of patients diagnosed with obstructive sleep apnoea (OSA) are currently untreated or non-adherent to continuous positive airway pressure (CPAP) [1,2]. Thus, novel approaches to OSA therapy are required. In the last few years, the concept of personalised OSA therapy has emerged, based on the idea that OSA interventions have maximal impact when they match patients' underlying pathophysiology or "endophenotypes" [3-7]. At the core of this notion is the recognition that OSA emerges as the consequence of different endophenotypic traits in different individuals, namely 1) increased pharyngeal collapsibility, 2) reduced ventilatory control stability (elevated loop gain, i.e. an exaggerated ventilatory drive response to reduced airflow and attendant hypoxia/hypercapnia), 3) reduced respiratory arousal threshold (a small rise in ventilatory drive terminates sleep) and 4) reduced compensatory pharyngeal dilator muscle activation [8-11].

A major hurdle for clinical implementation of personalised medicine is that the assessment of traits causing OSA has been confined to research laboratories $[9,12]$. To overcome this barrier, we recently developed an automated technique for estimating the four key traits [13-15] using routine clinical sleep studies (polysomnography).

Here, we prospectively tested the predictive value of phenotyping using polysomnography in a study of supplemental inspired oxygen, a therapy which acts specifically to lower loop gain [16] and substantially improves OSA in a subgroup of patients [3]. We tested the primary hypothesis that elevated loop gain, measured from clinical polysomnography, predicts a preferential reduction in OSA severity with a single night of supplemental oxygen (40\% inspired) versus sham (air) in a randomised single-blind crossover study (NCT01751971). We also assessed the predictive value of elevated loop gain in combination with the other three traits (post hoc analysis).

\section{Methods}

Participants

Patients with a previous clinical diagnosis of OSA with an apnoea-hypopnoea index (AHI) $>20$ events $\cdot \mathrm{h}^{-1}$ were eligible to participate. Patients using respiratory stimulants or depressants (including opioids and benzodiazepines) were excluded, as were those with diagnoses of heart failure or lung diseases, those with central rather than obstructive sleep apnoea (majority central respiratory events) and women who were pregnant. Participants provided written informed consent, and approval was granted by the Partners' Institutional Review Board.

We enrolled 47 participants; eight did not exhibit OSA and three did not attend the second overnight session, leaving 36 patients who completed the protocol (supplementary figure S1).

\section{Procedure}

Patients completed two overnight polysomnographic studies, one week apart (randomised order). Supplemental oxygen or medical air (sham) was delivered via a Venturi mask (40\% inspired oxygen, equivalent to $\sim 4 \mathrm{~L} \cdot \mathrm{min}^{-1}$ via a cannula [17]; see [3, 4]). The single-blind design enabled real-time monitoring of appropriate inspired oxygen levels $\left(\mathrm{O}_{2}\right.$ analyzer 17625; VacuMed, Ventura, CA, USA). Patients slept predominantly supine to minimise position effects. Supine blood pressure was measured (Dinamap Pro 100v2; GE Medical Systems, Tampa, FL, USA) during quiet wakefulness immediately preceding lights out (after $\geqslant 1 \mathrm{~h}$ of rest during setup and $\sim 10 \mathrm{~min}$ in the supine position) and again $\sim 10 \mathrm{~min}$ after lights on with oxygen/sham removed. We assessed self-reported sleep quality at study completion ("better", "same" or "worse" versus previous study; scored 1,0 and -1 , respectively) and alertness (Stanford Sleepiness Scale) each morning.

\section{Polysomnography}

Standard clinical polysomnographic instrumentation was used [18]. Airflow was assessed with nasal pressure and an oronasal thermistor. We prioritised recording of high-quality nasal pressure signals. Hypopnoeas were scored based on a 30\% reduction in airflow without oxygen desaturation or an arousal criterion (supplemental oxygen would otherwise mask hypopnoeas).

\section{Quantifying the pathophysiological traits using polysomnography}

Sham night polysomnography was used to quantify the baseline OSA traits using an automated method [13-15] described below.

\section{Chemical drive}

Phenotypic traits were quantified by first estimating "ventilatory drive", i.e. intended ventilation, using a chemoreflex feedback control model (gain, response time, delay, arousal response) fit to ventilation data [13]. 
Briefly, nasal pressure (square-root transformed) provided a ventilatory flow surrogate that was integrated to yield a breath-to-breath ventilation signal (uncalibrated tidal volumexrespiratory rate, meannormalised). 7 -min windows containing $\geqslant 1$ respiratory event were identified (non-rapid eye movement $(\mathrm{REM})$ ), and estimated ventilatory drive (model output) was best fit to the ventilation signal between obstructive events (when the airway was patent).

Loop gain

For each window, loop gain was calculated from the feedback model; the median was used to represent the night [13]. Two parameters were quantified: $\mathrm{LG}_{1}$ (a priori predictor) is the ventilatory drive response to a 1 cycle $\min ^{-1}$ reduction in ventilation and reflects "hypersensitivity" (e.g. increased chemosensitivity or reduced lung volume). $\mathrm{LG}_{\mathrm{n}}$ quantifies "instability" and also includes circulatory delay effects $\left(\mathrm{LG}_{\mathrm{n}}>1.0\right.$ yields periodic central apnoeas) $[3,19]$.

Arousal threshold

The arousal threshold was taken as the median estimated ventilatory drive preceding scored electroencephalogram arousals [15].

Upper airway physiology

Pharyngeal collapsibility ( $V$ passive) was defined here as the ventilation at normal/eupnoeic ventilatory drive $[9,12]$ during sleep and was quantified using an overnight breath-by-breath summary plot of ventilation versus estimated ventilatory drive [14]. Pharyngeal muscle "compensation" is the increase in ventilation from $V$ passive to the value at the arousal threshold ( $V$ active; compensation=Vactive $-V_{\text {passive). Greater }} V_{\text {passive }}$ and compensation indicate better pharyngeal patency and were expected to predict successful oxygen therapy.

\section{Definition of response to therapy}

Patients were considered "responders" if their AHI was reduced by $\geqslant 50 \%$ with treatment versus sham (a priori criterion); patients were otherwise considered "non-responders". Responses were considered "complete" if the AHI was also $<15$ events $\cdot \mathrm{h}^{-1}$ with treatment [4], equivalent to a $>67 \%$ reduction in AHI in our population. Non-responders were considered "borderline" if they exhibited a $>33 \%$ reduction in AHI.

\section{Assessment of predictive value}

The predictive value of loop gain and other traits was assessed based on whether there was a significant difference in the reduction in AHI (as a percentage of the baseline value) between defined "predicted responder" and "predicted non-responder" subgroups (e.g. high versus low loop gain). The pre-specified choice of loop gain parameter $\left(\mathrm{LG}_{1}\right)$ and a cut-off of 0.7 (i.e. the threshold that defines high versus low $\mathrm{LG}_{1}$ ) were based on prior findings [13]. For all post hoc subgroup classification tests, including multivariable analysis (below), cut-off values were selected to maximise sensitivity plus specificity [20]. Predictive values were estimated using leave-one-out cross-validation, whereby each subject's response was predicted using a modified version of the same model with the subject's own data left out.

\section{Multivariable model analysis}

We employed logistic regression using the phenotypic variables, i.e. loop gain, collapsibility, arousal threshold and muscle compensation, to define subgroups of predicted responders and predicted non-responders; a quadratic model that included interaction terms and squared terms was chosen based on clear evidence of interactions. Terms were selected using backwards elimination ( $p$-to-remove $=0.157$ ) [21]. To raise statistical power, we included training data from a previous study employing supplemental oxygen $(n=20)$ to help build a more robust regression model [4]; the predictive value is reported exclusively for patients in the current study (supplementary material).

\section{Statistical analysis}

Unpaired t-tests or Wilcoxon rank-sum tests were used to compare differences between groups (responders versus non-responders; predicted responders versus predicted non-responders). Standard errors for proportions (positive/negative predictive values, i.e. proportion of correct predictions given a positive/negative prediction) employed the normal approximation to the binomial distribution; $\mathrm{p}$-values indicate differences from chance values. Significance was accepted at $\mathrm{p}<0.05$.

\section{Results}

Baseline characteristics are detailed in table 1. Supplemental oxygen lowered AHI by $\sim 30 \%$ overall (supplementary table S1) confirmed by an $\sim 25 \%$ reduction in arousal index. Responders ( $\mathrm{n}=9$ ) exhibited an $\sim 70 \%$ reduction in AHI, accompanied by an $\sim 50 \%$ reduction in the frequency of arousals from sleep, an $\sim 50 \%$ reduction in the time spent in light sleep (stage 1 non-REM), and an $\sim 7 \mathrm{mmHg}$ reduction in the 


\section{TABLE 1 Patient characteristics}

\begin{tabular}{|c|c|c|c|c|}
\hline Characteristic & All patients & Responders" & Non-responders & p-value ${ }^{\pi}$ \\
\hline Subjects & 36 & 9 & 27 & \\
\hline \multicolumn{5}{|l|}{ Demographics } \\
\hline Male/female & $26 / 10$ & $6 / 3$ & $20 / 7$ & 0.7 \\
\hline Race & & & & 0.015 \\
\hline Black & 9 & 5 & 4 & \\
\hline Other & 1 & 0 & 1 & \\
\hline Body mass index $\mathrm{kg} \cdot \mathrm{m}^{-2}$ & $31.1 \pm 0.7$ & $32.3 \pm 1.2$ & $30.6 \pm 0.8$ & 0.3 \\
\hline Neck circumference $\mathrm{cm}$ & $40.6 \pm 0.5$ & $40.2 \pm 1.0$ & $40.7 \pm 0.7$ & 0.7 \\
\hline Systolic blood pressure ${ }^{+} \mathrm{mmHg}$ & $136.8 \pm 2.4$ & $134.9 \pm 3.7$ & $137.5 \pm 2.9$ & 0.6 \\
\hline Diastolic blood pressure ${ }^{+} \mathrm{mmHg}$ & $80.7 \pm 1.9$ & $79.7 \pm 3.8$ & $81.1 \pm 2.2$ & 0.8 \\
\hline Current use of anti-hypertensive medication $\mathrm{n}(\%)$ & 12 (33) & $1(11)$ & $11(41)$ & 0.2 \\
\hline \multicolumn{5}{|l|}{ Polysomnography ${ }^{\S}$} \\
\hline $\mathrm{AHI}$ events $\cdot \mathrm{h}^{-1}$ & $57.9 \pm 22.1$ & $56.6 \pm 7.7$ & $58.3 \pm 4.3$ & 0.8 \\
\hline Central events ${ }^{f}$ & $4.7 \pm 2.0$ & $8.5 \pm 5.7$ & $3.4 \pm 1.8$ & 0.3 \\
\hline Hypopnoeas $^{f}$ & $47.3 \pm 5.4$ & $61.9 \pm 11.3$ & $42.5 \pm 6.0$ & 0.12 \\
\hline Arousals ${ }^{f}$ & $88.8 \pm 4.9$ & $79.1 \pm 5.2$ & $92.1 \pm 6.2$ & 0.3 \\
\hline Nadir oxygen saturation $\%$ & $87.1 \pm 4.8$ & $89.2 \pm 1.5$ & $86.4 \pm 0.9$ & 0.13 \\
\hline Stage 1 sleep as $\%$ total sleep time & $25.9 \pm 22.0$ & $22.3 \pm 5.1$ & $27.1 \pm 4.6$ & 0.6 \\
\hline \multicolumn{5}{|c|}{$\begin{array}{l}\text { Data are presented as } \mathrm{n} \text { or mean } \pm \mathrm{SEM} \text {, unless otherwise indicated. CPAP: continuous positive airway pressure; AHI: apnoea-hypopnoea index. } \\
\# \text { : responders are defined by a } \geqslant 50 \% \text { reduction in } \mathrm{AHI} \text {; }{ }^{\text {? }} \text { : } \mathrm{t} \text {-tests were used for continuous variables and Fisher exact tests were used for } \\
\text { categorical variables (including black versus not black, treated versus not treated); }{ }^{+} \text {: morning, supine, off treatment (sham night); } \\
\S \text { : polysomnography refers to the sham night; respiratory event and oxygenation data reflect supine non-random eye movement sleep; }{ }^{f} \text { : data } \\
\text { presented as } \% \text { of respiratory events. }\end{array}$} \\
\hline
\end{tabular}

overnight change in blood pressure (figure 1). These changes were not observed in non-responders $(\mathrm{n}=27)$. Six of nine responders also had an AHI of $<15$ events $\cdot \mathrm{h}^{-1}$ on oxygen (i.e. complete responders). Overall, most patients felt they slept "better" on the oxygen night (better:same:worse on oxygen versus air=19:9:7). There was no effect on subjective alertness (change in Stanford Sleepiness Scale rating: +0.1 \pm 0.2 units, higher value represents reduced alertness).

\section{Loop gain and responses to oxygen}

Example measurements in one responder and one non-responder are shown in figure 2. Contrary to our primary hypothesis, elevated baseline loop gain was not a significant univariate predictor of the response to treatment (\% reduction in AHI with supplemental oxygen versus sham), i.e. there was no difference in the response between patients with higher versus lower loop gain based on $\mathrm{LG}_{1}(35.7 \pm 6.6 \%$ versus 25.2 $\pm 9.0 \%, p=0.4$; pre-specified cut-off $=0.7$; supplementary figure S2). However, there was a strong trend towards a greater response in those with higher loop gain based on $\mathrm{LG}_{\mathrm{n}}$ (figure 3).

\section{Other physiological traits and responses to oxygen}

Reduced collapsibility (higher $V$ passive) and greater compensation were strong predictors of the response to oxygen, and there was a trend towards a greater response in patients with lower arousal threshold (figure 3).

\section{Multivariable model analysis}

When traits were considered in combination (multivariable logistic regression), a higher loop gain increased the likelihood of being a responder, particularly in patients with better compensation; poor compensation and poor collapsibility reduced the likelihood of being a responder (table 2, figure 4; see also supplementary table S2, supplementary figure S3). The regression model exhibited excellent predictive value overall $(\triangle \mathrm{AHI}$ in predicted responders versus predicted non-responders: $62 \pm 5 \%$ versus $10 \pm 7 \%, \mathrm{p}<0.0001$; positive predictive value $(\mathrm{PPV})=69 \pm 13 \%, \mathrm{p}=0.0005$; negative predictive value 

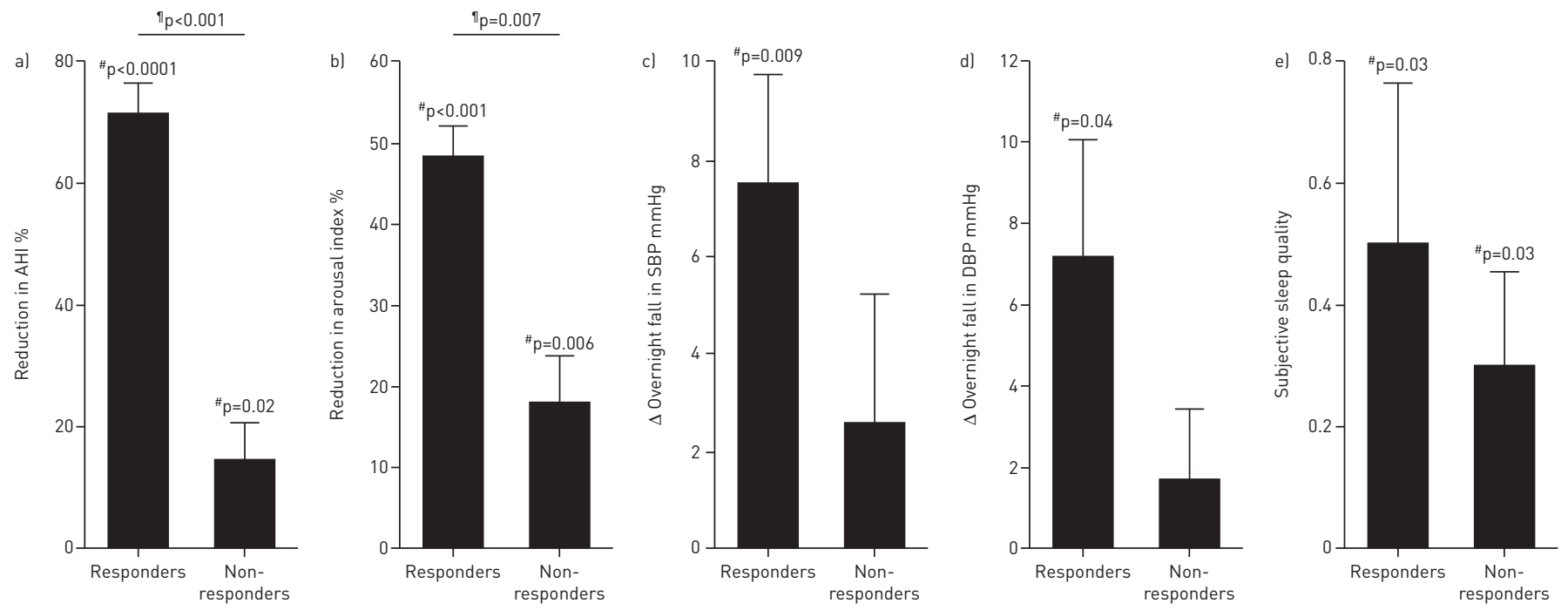

FIGURE 1 Effect of supplemental oxygen on primary and secondary outcomes in responders ( $\mathrm{n}=9$ ) and non-responders ( $\mathrm{n}=27$ ). a) In responders, improvements were observed in the apnoea-hypopnoea index (AHI) by definition. In addition, responders exhibited improvements in b) the frequency of arousals from sleep (arousal index) as well as in c, d) blood pressure (change from evening to morning) and e) subjective sleep quality. Subjective sleep quality was scored as follows: $1=$ slept better; $0=s l e p t$ the same; $-1=$ slept worse. There was no effect on the Stanford Sleepiness Scale (subjective morning alertness, not shown). Error bars indicate SEM. SBP: systolic blood pressure; DBP: diastolic blood pressure.

\#: oxygen versus sham; ": responders versus non-responders.

$(\mathrm{NPV})=100 \pm 0 \%, \mathrm{p}<0.0001 ;$ accuracy $=89 \pm 5 \%, \mathrm{p}<0.0001)$; after cross-validation results remained strong $(\Delta \mathrm{AHI}=58 \pm 6 \%$ versus $12 \pm 7 \%, \mathrm{p}=0.0001 ; \mathrm{PPV}=62 \pm 13 \%, \mathrm{p}=0.007 ; \mathrm{NPV}=96 \pm 4 \%, \mathrm{p}<0.0001 ;$ accuracy $=83$ $\pm 6 \%, \mathrm{p}=0.0002)$.

\section{Secondary outcomes in predicted responders}

Predicted responders (cross-validated results) exhibited improvements with oxygen in arousal index, blood pressure and subjective sleep quality (slept better, same or worse on oxygen versus air) that were not observed in predicted non-responders (figure 5). Stanford Sleepiness Scale ratings were unchanged in both subgroups.

\section{Predictive values of non-physiological variables}

Predicted responder/non-responder status based on patients' pathophysiology remained significantly associated with responses to oxygen ( $p=0.002-0.003$ ) after adjusting for common clinical factors (age, sex, body mass index, neck circumference, current CPAP use and AHI off treatment; logistic regression with covariates added separately; supplementary figure S4). These clinical factors were not significant predictors.

\section{Effect of oxygen on the physiological traits}

To understand whether oxygen therapy adversely affected traits other than loop gain, particularly in non-responders, we also assessed the effect of oxygen on the traits causing sleep apnoea (table 3; individual data given in supplementary figure S5). As expected [13, 16], oxygen lowered loop gain (instability; $\mathrm{LG}_{\mathrm{n}}$ ) through a reduction in ventilatory control sensitivity $\left(\mathrm{LG}_{1}\right)$ but had no influence on collapsibility ( $V$ passive) or compensation. However, oxygen lowered our measure of the arousal threshold. Effects were similar between responder and non-responder subgroups.

\section{Discussion}

The current study demonstrated that quantifying the pathophysiological traits of OSA patients using diagnostic polysomnography can identify patients whose condition is likely to be treatable using supplemental oxygen, and can rule out non-responders. In contrast to our specific hypothesis, elevated loop gain alone was not a strong predictor of the response to oxygen. Rather, elevated loop gain in combination with greater pharyngeal patency (less-severe collapsibility and greater compensation) predicted improved OSA; the combined multivariable model accurately ruled out a positive response to treatment (95\% certainty), and identified a subgroup of predicted responders who 1) had a $62 \%$ likelihood of halving the frequency of respiratory events and a $46 \%$ likelihood of adequately treating OSA $\left(\mathrm{AHI}<15\right.$ events $\left.\cdot \mathrm{h}^{-1}\right)$, and 2 ) experienced a significant reduction in morning blood pressure (relative to evening values) and an improvement in self-reported sleep quality (slept better than on the night without 
a)

Responder

Arousal

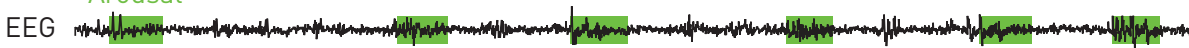

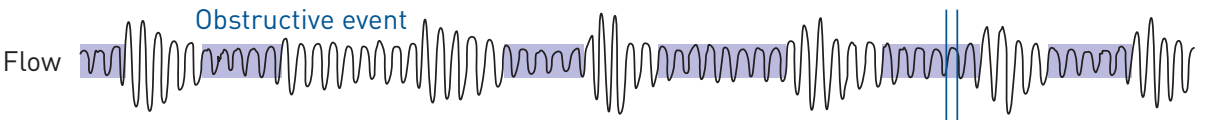

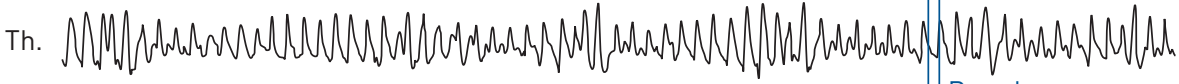

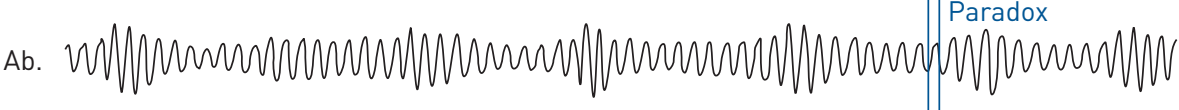

Ventilation \%

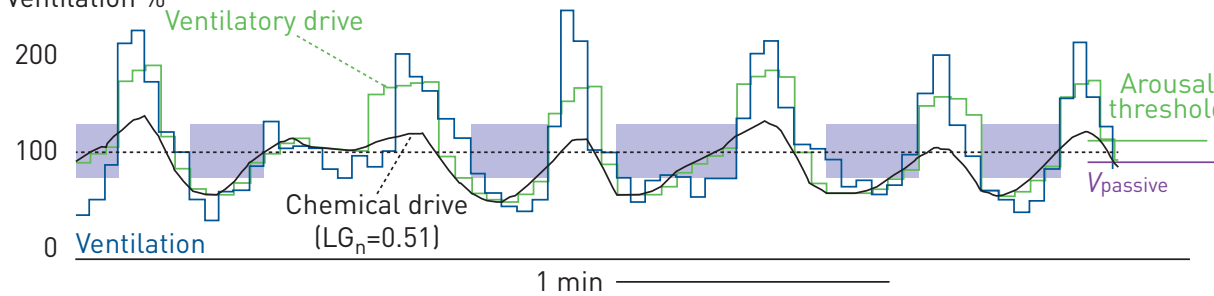

c)

Non-responder

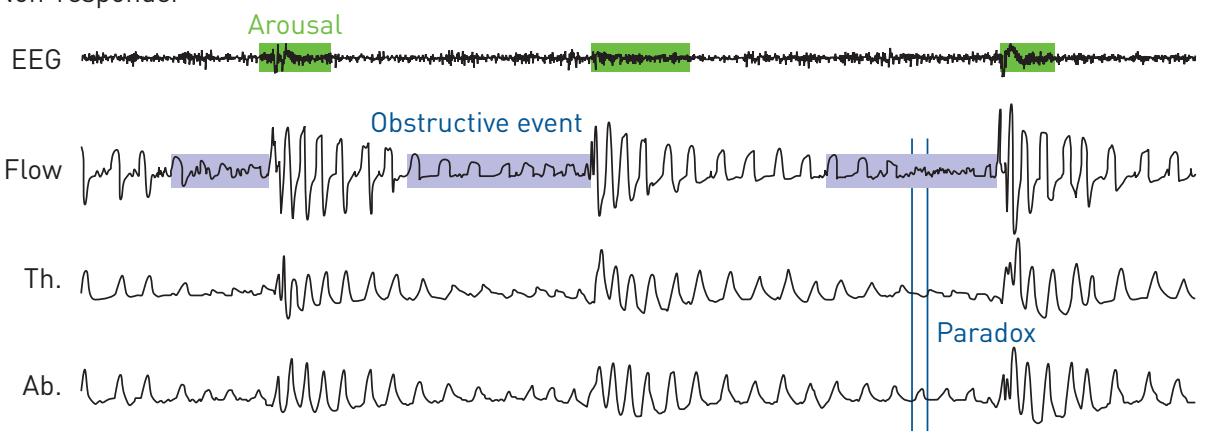

Ventilation \%

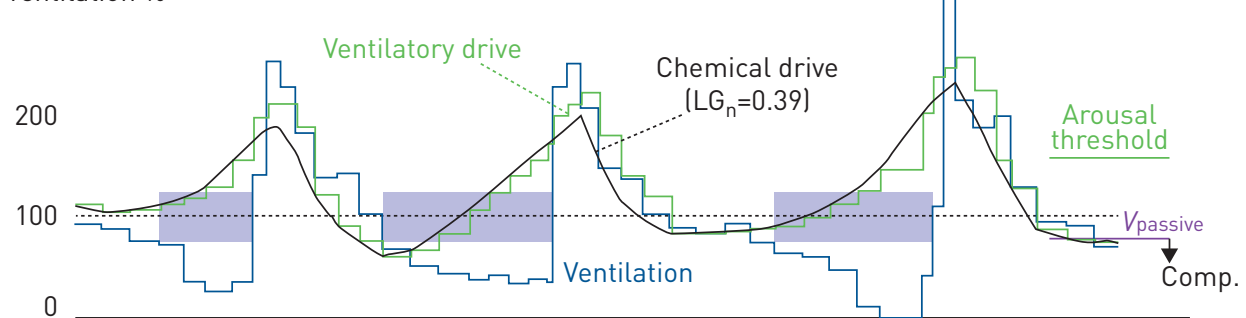

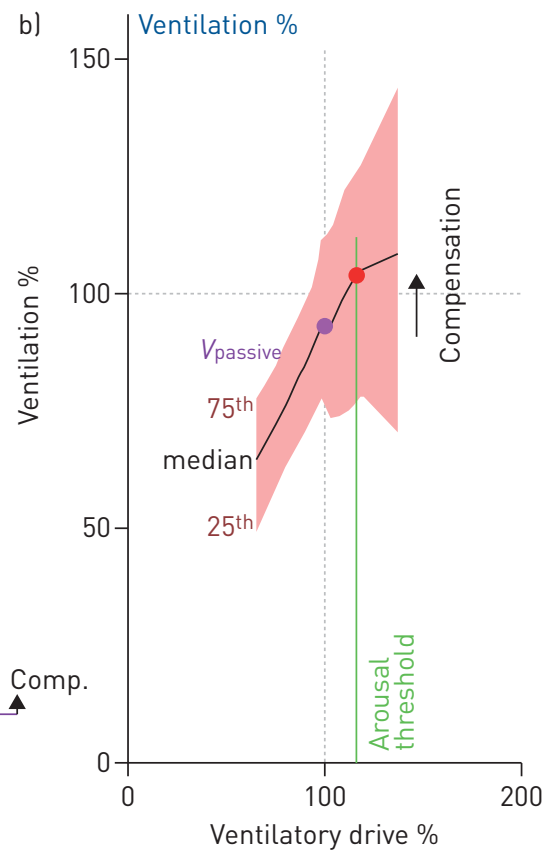

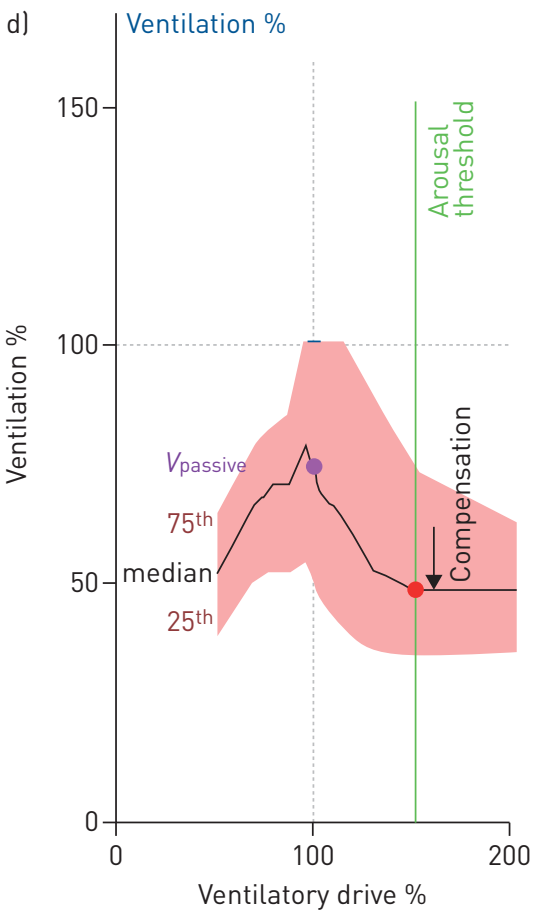

FIGURE 2 Example endophenotype data off treatment are shown for a, b) a responder (sham apnoea-hypopnoea index (AHI)=44.9, treatment $A H I=9.7$ events $\cdot h^{-1}$ ) and c, d) a non-responder (sham $A H I=42.8$, treatment $A H I=45.2$ events $\cdot h^{-1}$ ). a, c) Illustrative traces of sleep apnoea and model estimation of ventilatory drive. Note that events are self-similar within a subject. In the responder, changes in ventilation track estimated ventilatory drive during obstructive events. By contrast, in the non-responder ventilation falls as ventilatory drive rises. In the model estimations of ventilatory drive, note that the thoracic (Th.) and abdominal (Ab.) excursion (piezoelectric respiratory belts) signals are out of phase (paradox) during events in both subjects, consistent with airflow obstruction. Ventilation and ventilatory drive are expressed as a proportion of the mean ventilation during the

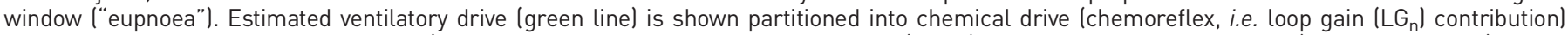
and the ventilatory response to arousal (arousal contribution, green minus black line). b, d) Summary plots of ventilation (i.e. actual airflow) versus ventilatory drive (i.e. intended airflow) during sleep (black line: median; shading: interquartile range). The responder has a higher loop gain, a lower ventilatory drive preceding arousal (arousal threshold) and less-severe collapsibility as inferred from the higher level of ventilation at normal ventilatory drive ( $V_{\text {passive }}$. EEG: electroencephalogram; Flow: square-root transformed nasal pressure; Comp: compensation.

treatment). Our study shows that measuring and combining key endophenotypic causes of sleep apnoea, based on a routine sleep study, provides insight into the response to an intervention and opens the door for personalising intervention based on underlying mechanisms. 
Responders

- Complete responders $(\triangle \mathrm{AH} \mathrm{H} \geqslant 67 \%)$

- Borderline responders $(50 \% \leqslant \Delta \mathrm{AH} \mid<67 \%)$
Non-responders

- Complete non-responders $(\triangle \mathrm{AH} \mid<33 \%)$

- Borderline non-responders $(33 \% \leqslant \Delta \mathrm{AH} \mid<50 \%)$
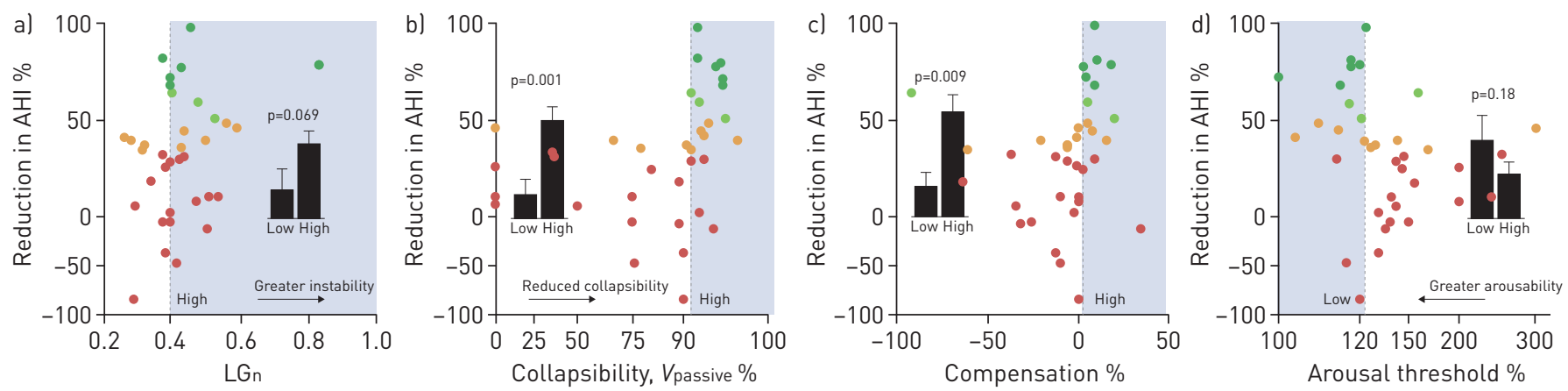

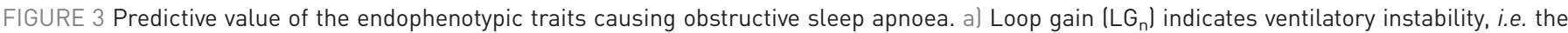
predisposition to spontaneous periodic breathing. b) Collapsibility (Vpassive), c) compensation and d) arousal threshold data are presented as a proportion of eupnoeic levels. See text for details. Shading illustrates the region of predicted responders and definition of high versus low for each trait subgroup. Bars illustrate the reduction in apnoea-hypopnoea index (AHI) with treatment in the high versus low subgroups (mean \pm SEM, patients were assigned to subgroups using cross-validation). Note the y-axis scale is compressed below zero to facilitate visual interpretation of values above zero. Each trait had significant negative predictive value: $\mathrm{LG}_{\mathrm{n}}$ (reduction in $\mathrm{AHI}$ : $37.6 \pm 6.9 \%$ versus $14.0 \pm 11.5 \%$; positive predictive value $(P P V)=35 \pm 10 \%, p=0.3$; negative predictive value $(N P V)=92 \pm 7 \%, p=0.02) ;$ passive $(49.3 \pm 6.9 \%$ versus $10.9 \pm 8.1 \% ; P P V=47 \pm 12 \%, p=0.07 ; N P V=95$ $\pm 5 \%, p<0.001)$; compensation $(53.0 \pm 8.9 \%$ versus $18.5 \pm 7.2 \%$; PPV $=55 \pm 15 \%, p=0.049 ; N P V=88 \pm 6 \%, p=0.046)$; arousal threshold (39.7 $\pm 12.8 \%$ versus $22.2 \pm 6.0 \% ; P P V=50 \pm 13 \%, p=0.06 ; N P V=91 \pm 6 \%, p=0.009)$.

\section{Physiological insight \\ Elevated loop gain}

We hypothesised that elevated loop gain would predict the response to lowering loop gain with supplemental oxygen, based on the notion that patients with higher loop gain have the greatest range for lowering loop gain and improving OSA. For example, many forms of central sleep apnoea, a high loop gain condition, can be effectively treated with supplemental oxygen [22, 23]. In addition, our prior study $(\mathrm{n}=12)$ in OSA found that oxygen is more effective in patients with higher versus lower loop gain $[3,13]$. Yet here we found that loop gain alone was insufficient to predict the response to supplemental oxygen, confirming more recent studies illustrating that loop gain (or chemosensitivity) by itself is not strongly associated (or even inversely associated [24]) with the reduction in OSA severity with treatments that lower loop gain (e.g. oxygen, acetazolamide) $[4,6,25]$. The current study demonstrates that successfully targeting an abnormal pathophysiological trait for OSA treatment requires not just knowledge of the trait itself but also the other determinants of OSA.

\section{Greater pharyngeal patency}

Importantly, our study demonstrates that more-severe pharyngeal collapsibility and poor muscle compensation (as measured using polysomnography) rule out a positive OSA response to supplemental

TABLE 2 Logistic regression model for predicting responses to oxygen therapy

\begin{tabular}{lrrcll} 
Variable & $\boldsymbol{\beta}$ & SEM & Odds ratio $^{\#}$ & p-value & Interpretation \\
\hline Constant & -1.97 & 1.02 & & 0.01 & \\
Loop gain & 15.41 & 7.40 & 3.7 & 0.038 & Higher loop gain $\rightarrow$ success \\
Vpassive & 5.27 & 3.71 & 4.8 & 0.15 & Reduced collapsibility $\rightarrow$ success \\
Compensation & 15.09 & 6.62 & 45.5 & 0.023 & Greater compensation $\rightarrow$ success \\
Vpassivexcompensation & -58.53 & 29.97 & 0.11 & 0.036 & Poor collapsibility and poor compensation $\rightarrow$ failure \\
Loop gainxcompensation & -80.34 & 34.16 & 0.17 & 0.019 & Low loop gain and poor compensation $\rightarrow$ failure \\
Arousal thresholdxcompensation & -86.43 & 29.53 & 0.012 & 0.003 & Low arousal threshold and higher compensation $\rightarrow$ success \\
& & & & &
\end{tabular}

Data represent the final regression results (six terms) after backwards stepwise elimination ( $p$-to-remove $=0.157$ ) which began with four traits, their squares and all interaction terms (full quadratic model, $n$ terms=14). \#: odds ratio describes the increase in likelihood of being a responder per SD increase in each term. Traits were mean-subtracted before application to the regression model: mean $V_{\text {passive }}=62.8 \%$, mean loop gain $\left(L G_{n}\right)=0.42$, mean arousal threshold* $=157.6 \%$, mean compensation $=6.1 \%$. ${ }^{*}$ To promote normality, $V_{\text {passive }}$ and arousal threshold values were square-root transformed around $100 \%$ using $y=1+(x-1)^{0.5}$ and $y=1-(1-x)^{0.5}$ respectively $(x=1$ describes $100 \%)$; asterisks indicate transformed variables. Patients were considered a predicted responder if $Y=-1.97+15.41$ (loop gain) $+5.27\left(V_{\text {passive }}\right.$ ) +15.09 (compensation) -58.53 (Vassive* $\times$ compensation)-80.34(loop gain $\times$ compensation)-86.43(arousal threshold*-compensation)> -0.66 (use of this equation requires transformed, mean-subtracted traits). A simplified two-trait model is provided in the supplementary material (table S2, figure S3). 
Responders

- Complete responders $(\triangle \mathrm{AH} I \geqslant 67 \%)$

- Borderline responders $(50 \% \leqslant \Delta \mathrm{AH} \mid<67 \%)$
Non-responders

- Complete non-responders $(\triangle \mathrm{AH} \mathrm{H}<33 \%)$

- Borderline non-responders $(33 \% \leqslant \Delta \mathrm{AH} \mid<50 \%)$ a)
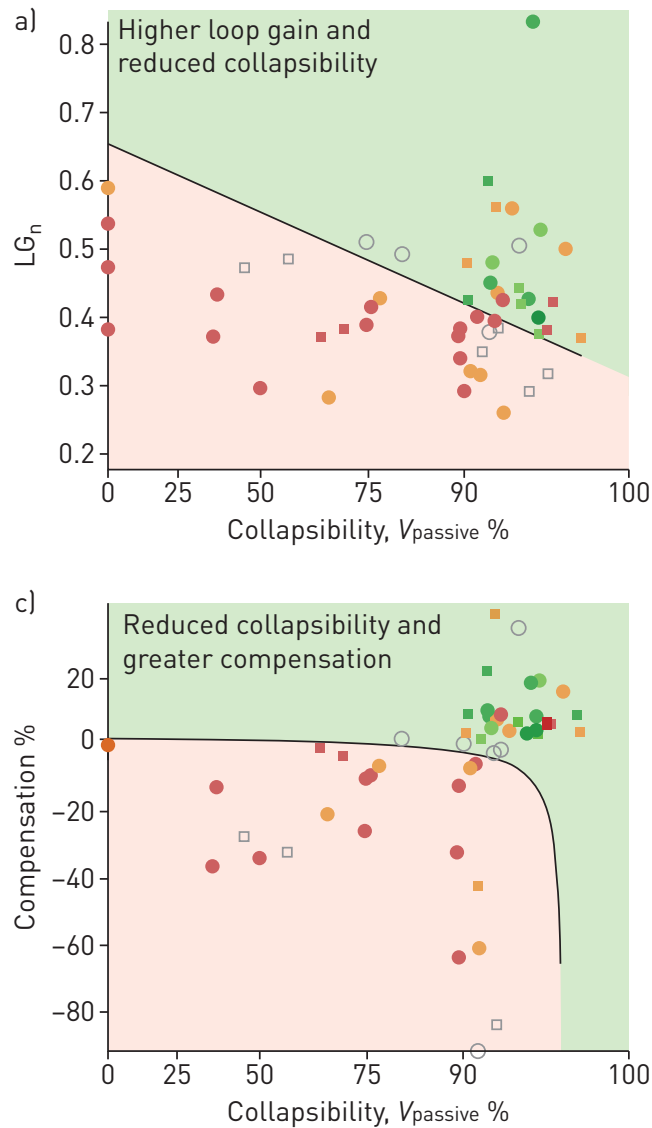
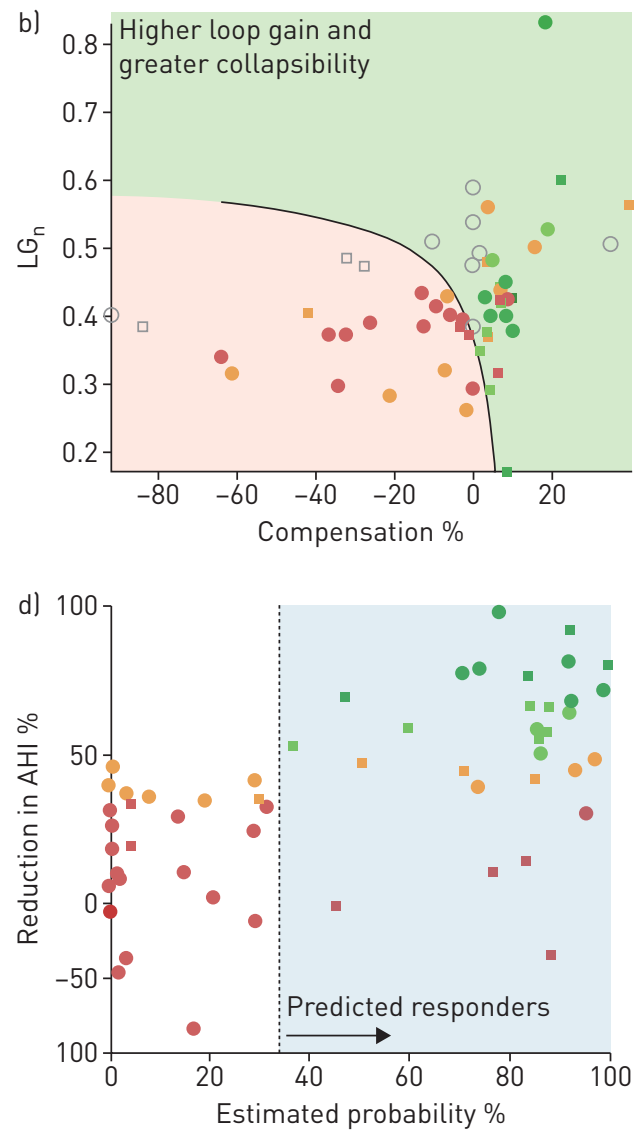

FIGURE 4 Multivariable analysis of the obstructive sleep apnoea traits. a-c) Two-trait "slices" of the four-trait regression model illustrate how the traits causing sleep apnoea combine to predict responses to supplemental oxygen. Dots are individual patients (circles are patients from current study, squares are patients from EDWARDS et al. [4]). Shading illustrates the regions of "predicted responders" (green) and "predicted non-responders" (red). Each two-trait slice represents model predictions at constant values of the other two traits; data points that are far enough away from the slice such that the slice prediction does not match the overall model prediction (irrespective of correct/incorrect) are shown in light grey. d) The continuous relationship between the reduction in apnoea-hypopnoea index (AHI) with oxygen and the regression model prediction is shown ( probability $=1 /\left(1+e^{-Y}\right)$; see table 2$)$. Note the $y$-axis scale is compressed below zero to facilitate visual interpretation of values above zero.

oxygen. These findings are consistent with OSA pathophysiology; patients who have both a high loop gain and reduced airway patency will continue to have reduced patency once loop gain is lowered, promoting residual OSA regardless of how high loop gain was at baseline $[4,16]$.

\section{Baseline OSA severity}

Remarkably, in the nine responders (25\% of patients), oxygen lowered the AHI from an average of 57 to 18 events.h ${ }^{-1}$ ( $\sim 70 \%$ reduction), illustrating that supplemental oxygen can have a large impact in the right patients even in severe OSA. Responders were not identified by milder sleep apnoea at baseline, i.e. a lower AHI. Thus, OSA severity is unlikely to contribute meaningfully to clinical decisions regarding the use of loop-gain-lowering interventions for OSA (table 1, supplementary table S1, supplementary figure S4).

\section{Ruling out versus ruling in}

Polysomnographic phenotyping accurately ruled out non-responders to supplemental oxygen (high NPV), but was somewhat less accurate at ruling in responders to treatment. Thus, it remains uncertain whether a patient with appropriate pathophysiology will respond favourably to treatment. A more certain outcome might require that the intervention also consistently succeed at improving the pathophysiology (lowering loop gain) across patients, but the magnitude of such improvements vary considerably [3, 4, 13, 25]. 


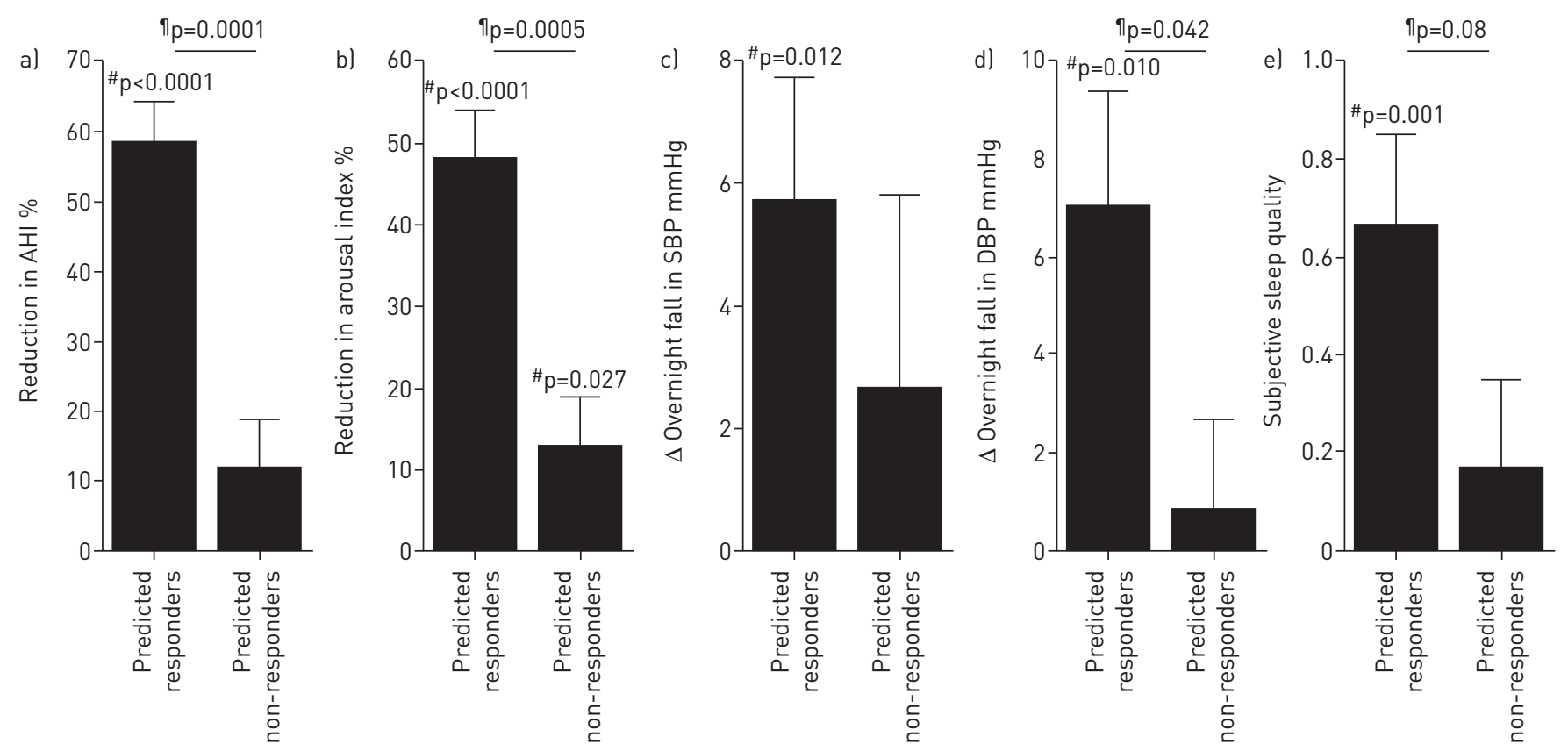

FIGURE 5 Effect of supplemental oxygen on primary and secondary outcomes in patients with suitable pathophysiology, i.e. "predicted responders" ( $n=13)$, and patients with unsuitable pathophysiology, i.e. "predicted non-responders" (n=23), based on endophenotypic traits (logistic regression, cross-validated; table 2, figure 4). a) In predicted responders, treatment led to an improvement in obstructive sleep apnoea severity (reduction in apnoea-hypopnoea index (AHI)); in contrast to figure 1, differences between predicted responders and non-responders are not "by definition" because subgroups were assigned using only data from the other subjects (i.e. cross-validation). Predicted responders also exhibited improvements in b) the frequency of arousals from sleep, c, d) blood pressure (evening minus morning levels) and e) subjective sleep quality. Subjective sleep quality was scored as follows: $1=$ slept better; $0=$ slept the same; $-1=$ slept worse. There was no effect on the Stanford Sleepiness Scale (subjective morning alertness, not shown) in either subgroup. SBP: systolic blood pressure; DBP: diastolic blood pressure. ${ }^{\#}$ : oxygen versus sham; ${ }^{\text {ๆ }}$ : responders versus non-responders. Compare results with figure 1.

Regardless, by ruling out clear non-responders, our method potentially allows clinicians and investigators to limit future trials of supplemental oxygen (or other agents lowering loop gain) to the subgroup of OSA patients with the most favourable pathophysiology.

TABLE 3 Effect of oxygen therapy on the physiological traits

Phenotypic trait

Overall effects $(n=36)$

Responders ( $\mathrm{n}=9$ ) versus non-responders $\left(\mathrm{n}=26^{\#}\right)$

\begin{tabular}{|c|c|c|c|c|c|c|c|}
\hline & Sham & Oxygen therapy & Median change & p-value ? & $\begin{array}{l}\text { Median change } \\
\text { within } \\
\text { responders }\end{array}$ & $\begin{array}{l}\text { Median change } \\
\text { within } \\
\text { non-responders }\end{array}$ & p-value ${ }^{+}$ \\
\hline $\begin{array}{l}\text { Loop gain, } \text { LG }_{1} \\
\text { (sensitivity) }\end{array}$ & 0.63 (0.56 to 0.75$)$ & $0.46(0.40$ to 0.58$)$ & $-0.18(-0.25$ to -0.04$)$ & $<0.00001$ & $\begin{array}{c}-0.20^{*} \\
(-0.40 \text { to }-0.10)\end{array}$ & $\begin{array}{c}-0.17^{* * *} \\
(-0.24 \text { to }-0.03)\end{array}$ & 0.4 \\
\hline $\begin{array}{l}\text { Arousal } \\
\text { threshold \% }\end{array}$ & $131(116$ to 151$)$ & 118 (109 to 150$)$ & $-9.8(-20$ to 2$)$ & 0.038 & $\begin{array}{c}-9.8^{*} \\
(-21.6 \text { to }-2.2)\end{array}$ & $\begin{array}{c}-9.5 \\
(-17.4 \text { to } 10.7)\end{array}$ & 0.3 \\
\hline $\begin{array}{l}V_{\text {passive } \%} \\
\quad \text { (collapsibility }{ }^{\S} \text { ) }\end{array}$ & 91.5 (75.0 to 94.2 ) & $87.3(69.7$ to 94.8 ) & $0.0(-11.2$ to 4.0$)$ & 0.7 & $\begin{array}{c}+0.6 \\
(-0.7 \text { to } 3.8)\end{array}$ & $\begin{array}{c}-2.1 \\
(-13.4 \text { to } 4.0)\end{array}$ & 0.6 \\
\hline Compensation \% & $0(-11.8$ to 6.8$)$ & $-1.9(-22.5$ to 5.8$)$ & $-3.2(-13.1$ to 11.9$)$ & 0.5 & $\begin{array}{c}-4.7 \\
(-11.5 \text { to } 1.7)\end{array}$ & $\begin{array}{c}-1.6 \\
(-16.8 \text { to } 12.8)\end{array}$ & 0.9 \\
\hline
\end{tabular}

Data are presented as median (interquartile range). ": one non-responder provided no data; no 7-min windows of non-rapid eye movement

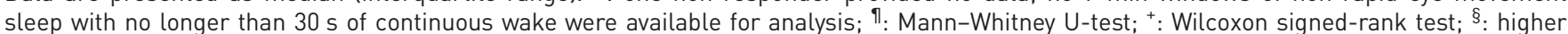

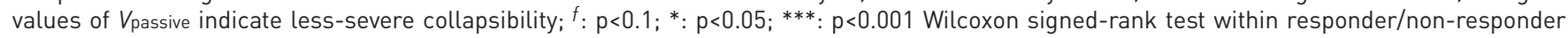
subgroups. 
Effect of oxygen therapy on the traits

We found that supplemental oxygen lowered our estimate of loop gain and had no influence on our estimates of upper airway physiology (collapsibility and compensation), consistent with previous data [16]; there was also no evidence of a differential effect on collapsibility or compensation in non-responders versus responders (table 2, supplementary figure S5) to support the notion that oxygen may worsen upper airway traits in non-responders. We also observed that oxygen lowered the arousal threshold, i.e. it had a deleterious effect. The direction of influence, and the clearer effect in responders, indicates that this effect is not a plausible cause of the improvement in OSA or of differences between responders and non-responders. Lowering of the arousal threshold may occur consequent to a habituation effect accompanying the amelioration of OSA [26]. This observation is also consistent with our previous finding that reducing inspired oxygen levels (hypoxia) increases the arousal threshold [16].

\section{Additional outcomes}

Supplemental oxygen was recently confirmed to have no impact on lowering blood pressure in unselected patients with OSA, in contrast to CPAP $[27,28]$. On the surface, those findings may appear to contrast with ours. However, in our study, blood-pressure-lowering effects (i.e. reduced morning minus evening values of systolic and diastolic blood pressure) were only exhibited in the subgroup that responded to treatment. Thus, the available evidence suggests that oxygen administration may exclusively lower blood pressure in the subgroup of patients in whom it also improves OSA. Longer-term investigations are warranted.

\section{Clinical implications}

A large proportion of OSA patients are intolerant of CPAP, and supplemental oxygen is currently used as a salvage therapy for OSA primarily to maintain nocturnal oxygenation. In unselected patients, improvements in oxygenation are consistent but improvements in OSA are modest $(\sim 10-30 \%$ reduction in AHI) [3, 6, 27-31]; in some individuals, oxygen may even increase OSA severity (figures 2-5). However, in patients with a specific set of phenotypic traits, supplemental oxygen can have clinically relevant effects $(>50 \%$ reduction in AHI; figures 4 and 5). In addition, a recent trial indicated that adherence to supplemental oxygen, while imperfect, is superior to CPAP [27], suggesting that supplemental oxygen may be well tolerated in these individuals.

Our approach to identifying likely responders was to quantify the pathophysiological traits causing OSA using clinical sleep studies and a technique that requires no invasive equipment or positive airway pressure manipulation and is fully automated. Thus, our approach can be readily implemented in the clinical setting.

The specific four-trait regression model (figure 4) was developed based on the available data (post hoc) and tested using a leave-one-out cross-validation approach, which makes efficient use of limited data. We emphasise that our specific model needs to be confirmed via prospective testing, preferably over a longer treatment period, to confirm clinical utility. Because our findings are consistent with physiological principles, we believe that the likelihood of reproducibility is high.

Our study also provides proof of principle that it is possible to identify OSA responders to loop-gain-lowering therapies more generally. We hope that our results will also apply to the prediction of responses to other current and future OSA interventions targeting loop gain, e.g. acetazolamide [25], $\mathrm{H}_{2} \mathrm{~S}$ inhibitors [32] and purinergic antagonists [33]. The general approach to combining noninvasive trait measurements may also help to predict responses to other therapies, such as pharyngeal surgery [34, 35]. Further investigations along these lines are needed.

\section{Methodological considerations}

In the current study, subjects were studied on one night of treatment with supplemental oxygen. Thus, possible additional improvements in OSA severity over time were not captured $[26,36]$. However, available data indicate that oxygen has no major impact on OSA severity beyond the effects on the first night, and observed improvements in OSA severity are immediately reversed with treatment discontinuation [30]. Nonetheless, longer-term studies are necessary to examine the effects of oxygen on OSA symptoms and blood pressure in predicted responders. For purposes of scientific veracity we administered supplemental oxygen through a Venturi mask at a moderate concentration ( $40 \%$ inspired, equivalent to $\sim 4 \mathrm{~L} \cdot \mathrm{min}^{-1}$ by nasal cannula), at a level used previously [3,4]. We thereby 1 ) avoided interference between nasal cannula airflow measurement and oxygen/sham administration, 2) avoided a titration procedure which may have affected patient blinding and reduced the proportion of the night on optimal therapy, and 3) minimised dosage differences that might have confounded assessment of outcomes between subgroups.

We interpreted the results of our multiple-trait model (including high loop gain and less-severe collapsibility) as evidence that there is an identifiable patient subgroup that responds preferentially to oxygen therapy. We also consider the possibility that this phenotype could simply have a form of less-severe OSA (albeit previously unrecognised based on AHI or other polysomnographic measures), which might be easier to treat in general 
regardless of the type of OSA intervention. While further studies are needed to demonstrate oxygen-therapy specificity, the available evidence refutes this notion. First, the predicted-responder subgroup exhibited selective improvements in blood pressure and self-reported sleep quality, demonstrating that the responder phenotype of OSA appears clinically important, i.e. is non-trivial. Second, higher loop gain measured using polysomnography has been shown to predict non-responders to pharyngeal surgery, illustrating that the high loop gain contribution to the oxygen-responder phenotype is not a biomarker of easier-to-treat OSA [35]. Third, our recent data suggest that higher loop gain and less-severe collapsibility (the oxygen-responder phenotype) predicts that oral appliance therapy will fail [37], suggesting that oxygen responders are likely to be non-responders to other therapies; indeed, high loop gain via CPAP manipulation also predicts oral appliance non-responders [5]. A study is underway to investigate this issue further (NCT03189173).

We note that the values for physiological traits obtained in the current study from routine polysomnography are surrogates and might differ from those obtained with invasive "gold standard" measures used in specialised physiology laboratories. However, our measures compare favourably with gold standard values (correlation coefficients $\sim 0.7$ for each trait) [13-15].

The primary outcome variable was the AHI measured in supine non-REM sleep, with respiratory events scored using modified criteria that avoid reliance on the oxygen saturation signal (respiratory events require $\geqslant 30 \%$ reduction in airflow). Use of standard criteria for scoring respiratory events $(\geqslant 30 \%$ reduction in flow with $\geqslant 3 \%$ desaturation or arousal), or inclusion of all sleep states and positions, yielded similar results (supplementary table $\mathrm{S} 1$ ).

Patients slept supine because this captures OSA pathophysiology at its worst; treatment of lateral OSA without mechanical intervention would have been a less formidable challenge. In a patient who sleeps entirely lateral, we see no reason that the same traits while lateral would not predict responses to oxygen therapy also in the lateral position. Additional testing is warranted.

\section{Conclusions}

For the first time, we show that measuring the pathophysiologic variables causing OSA from a clinical sleep study (off treatment) can predict which patients are most suitable for supplemental oxygen therapy. A multivariable model incorporating increased loop gain with an improved airway (better collapsibility and compensation) accurately predicted responders to therapy: predicted responders not only exhibited improvements in OSA severity, but also experienced improvements in blood pressure and perception of sleep quality. We consider that phenotyping in this manner will provide an avenue for personalising interventions for patients who are intolerant of CPAP and may otherwise remain untreated.

Author contributions: Conception and design: S.A. Sands, B.A. Edwards, P.I. Terrill, A. Malhotra, A. Wellman. Methods development: S.A. Sands, P.I. Terrill, A. Azarbarzin. Data collection: S.A. Sands, B.A. Edwards, C.M. de Melo, E.T. Smales. Analysis: S.A. Sands, L.B. Hess. All authors interpreted data, edited the manuscript for important intellectual content and approved the final draft.

Conflict of interest: S.A. Sands reports grants from NIH and AHA, during the conduct of the study; and personal fees from Cambridge Sound Management, Nox Medical and Merck, outside the submitted work. B.A. Edwards received a salary from Heart Foundation of Australia, during the conduct of the study. P.I. Terrill reports grants from Australian National Health and Medical Research Council (1064163), during the conduct of the study; and grants from Hull Family Donation, 2014, outside the submitted work. J.P. Butler has nothing to disclose. R.L. Owens reports personal fees for consultancy from Novartis, and honoraria and travel reimbursement from ResMed and Itamar Medical, outside the submitted work. L. Taranto-Montemurro reports grants from American Heart Association, personal fees from Novion Pharmaceuticals and Cambridge Sound management, and other from Apnimed, outside the submitted work. A. Azarbarzin has nothing to disclose. M. Marques has nothing to disclose. L.B. Hess has nothing to disclose. E.T. Smales has nothing to disclose. C.M. de Melo has nothing to disclose. D.P. White reports personal fees from Philips Respironics (Chief Scientific Officer), personal fees for consultancy from Night Balance, and personal fees from Apnicure (previously Chief Medical Officer), outside the submitted work. A. Malhotra relinquished all outside personal income in 2012 as an Officer of the American Thoracic Society. ResMed provided a philanthropic donation to University College San Diego in support of a sleep centre. A. Wellman reports grants from National Institutes of Health and Philips Respironics, during the conduct of the study; grants from Varnum Sleep and Breathing Solutions and Cambridge Sound Management, and personal fees from Bayer and Nox Medical, outside the submitted work; in addition, A. Wellman has a patent Airway and Airflow Factors issued.

Support statement: This work was supported by the American Heart Association (15SDG25890059 to S.A. Sands), the NIH (R01HL102321, R01HL128658, P01HL094307 and P01HL095491 to A. Wellman and S.A. Sands), the National Health and Medical Research Council of Australia (1053201 to S.A. Sands; 1035115 to B.A. Edwards; and 1064163 to P.I. Terrill, S.A. Sands, A. Wellman and B.A. Edwards), the R.G. Menzies Foundation (S.A. Sands), the American Thoracic Society Foundation (S.A. Sands) and the Heart Foundation of Australia (Future Leader Fellowship 101167; B.A. Edwards). A. Malhotra is principal investigator on NIH R01HL085188, K24HL132105, and T32HL134632, and co-investigator on R21HL121794, R01HL119201 and R01HL081823. This work was also supported by Harvard Catalyst (National Center for Research Resources and the National Center for Advancing Translational Sciences, NIH Award UL1TR001102). Funding information for this article has been deposited with the Crossref Funder Registry. 


\section{References}

1 Skinner T, McNeil L, Olaithe M, et al. Predicting uptake of continuous positive airway pressure (CPAP) therapy in obstructive sleep apnoea (OSA): a belief-based theoretical approach. Sleep Breath 2013; 17: 1229-1240.

2 Rotenberg BW, Murariu D, Pang KP. Trends in CPAP adherence over twenty years of data collection: a flattened curve. J Otolaryngol Head Neck Surg 2016; 45: 43.

3 Wellman A, Malhotra A, Jordan AS, et al. Effect of oxygen in obstructive sleep apnea: role of loop gain. Respir Physiol Neurobiol 2008; 162: 144-151.

4 Edwards BA, Sands SA, Owens RL, et al. The combination of supplemental oxygen and a hypnotic markedly improves obstructive sleep apnea in patients with a mild to moderate upper airway collapsibility. Sleep 2016; 39: 1973-1983

5 Edwards BA, Andara C, Landry S, et al. Upper-airway collapsibility and loop gain predict the response to oral appliance therapy in patients with obstructive sleep apnea. Am J Respir Crit Care Med 2016; 194: 1413-1422.

6 Xie A, Teodorescu M, Pegelow DF, et al. Effects of stabilizing or increasing respiratory motor outputs on obstructive sleep apnea. J Appl Physiol 2013; 115: 22-33.

7 Eckert DJ, Owens RL, Kehlmann GB, et al. Eszopiclone increases the respiratory arousal threshold and lowers the apnoea/hypopnoea index in obstructive sleep apnoea patients with a low arousal threshold. Clin Sci 2011; 120: 505-514.

8 Younes M. Role of respiratory control mechanisms in the pathogenesis of obstructive sleep disorders. J Appl Physiol 2008; 105: 1389-1405.

9 Wellman A, Edwards BA, Sands SA, et al. A simplified method for determining phenotypic traits in patients with obstructive sleep apnea. J Appl Physiol 2013; 114: 911-922.

10 Eckert DJ, White DP, Jordan AS, et al. Defining phenotypic causes of obstructive sleep apnea. Identification of novel therapeutic targets. Am J Respir Crit Care Med 2013; 188: 996-1004.

11 McGinley BM, Schwartz AR, Schneider H, et al. Upper airway neuromuscular compensation during sleep is defective in obstructive sleep apnea. J Appl Physiol 2008; 105: 197-205.

12 Wellman A, Eckert DJ, Jordan AS, et al. A method for measuring and modeling the physiological traits causing obstructive sleep apnea. J Appl Physiol 2011; 110: 1627-1637.

13 Terrill PI, Edwards BA, Nemati S, et al. Quantifying the ventilatory control contribution to sleep apnoea using polysomnography. Eur Respir J 2015; 45: 408-418.

14 Sands SA, Edwards BA, Terrill PI, et al. Phenotyping pharyngeal pathophysiology using polysomnography in patients with obstructive sleep apnea. Am J Respir Crit Care Med 2018; 197, 1187-1197.

15 Sands SA, Terrill PI, Edwards BA, et al. Quantifying the arousal threshold using polysomnography in obstructive sleep apnea. Sleep 2018; 41: zsx183.

16 Edwards BA, Sands SA, Owens RL, et al. Effects of hyperoxia and hypoxia on the physiological traits responsible for obstructive sleep apnoea. J Physiol 2014; 592, 4523-4535.

17 Wettstein RB, Shelledy DC, Peters JI. Delivered oxygen concentrations using low-flow and high-flow nasa cannulas. Respir Care 2005; 50: 604-609.

18 Rechtschaffen A, Kales A. A Manual of Standardized Terminology, Techniques and Scoring Systems for Sleep Stages of Human Subjects. Los Angeles, CA, UCLA Brain Information Service/Brain Research Institute, 1968.

19 Khoo MC, Kronauer RE, Strohl KP, et al. Factors inducing periodic breathing in humans: a general model. $J$ Appl Physiol 1982; 53: 644-659.

20 Youden WJ. Index for rating diagnostic tests. Cancer 1950; 3: 32-35.

21 Heinze G, Dunkler D. Five myths about variable selection. Transpl Int 2017; 30: 6-10.

22 Weintraub Z, Alvaro R, Kwiatkowski K, et al. Effects of inhaled oxygen (up to 40\%) on periodic breathing and apnea in preterm infants. J Appl Physiol 1992; 72: 116-120.

23 Javaheri S, Ahmed M, Parker TJ, et al. Effects of nasal O2 on sleep-related disordered breathing in ambulatory patients with stable heart failure. Sleep 1999; 22: 1101-1106.

24 Wang D, Wong KK, Rowsell L, et al. Predicting response to oxygen therapy in obstructive sleep apnoea patients using a 10-minute daytime test. Eur Respir J 2018; 51: 1701587

25 Edwards BA, Sands SA, Eckert DJ, et al. Acetazolamide improves loop gain but not the other physiological traits causing obstructive sleep apnoea. J Physiol 2012; 590: 1199-1211.

26 Loewen A, Ostrowski M, Laprairie J, et al. Determinants of ventilatory instability in obstructive sleep apnea: inherent or acquired? Sleep 2009; 32: 1355-1365.

27 Gottlieb DJ, Punjabi NM, Mehra R, et al. CPAP versus oxygen in obstructive sleep apnea. N Engl J Med 2014; 370: $2276-2285$

28 Norman D, Loredo JS, Nelesen RA, et al. Effects of continuous positive airway pressure versus supplemental oxygen on 24-hour ambulatory blood pressure. Hypertension 2006; 47: 840-845.

29 Smith PL, Haponik EF, Bleecker ER. The effects of oxygen in patients with sleep apnea. Am Rev Respir Dis 1984; 130: 958-963.

30 Gold AR, Schwartz AR, Bleecker ER, et al. The effect of chronic nocturnal oxygen administration upon sleep apnea. Am Rev Respir Dis 1986; 134: 925-929.

31 Loredo JS, Ancoli-Israel S, Kim EJ, et al. Effect of continuous positive airway pressure versus supplemental oxygen on sleep quality in obstructive sleep apnea: a placebo-CPAP-controlled study. Sleep 2006; 29: 564-571.

32 Del Rio R, Marcus NJ, Schultz HD. Inhibition of hydrogen sulfide restores normal breathing stability and improves autonomic control during experimental heart failure. J Appl Physiol 2013; 114: 1141-1150.

33 Pijacka W, Moraes DJ, Ratcliffe LE, et al. Purinergic receptors in the carotid body as a new drug target for controlling hypertension. Nat Med 2016; 22: 1151-1159.

34 Li Y, Ye J, Han D, et al. Physiology-based modeling may predict surgical treatment outcome for obstructive sleep apnea. J Clin Sleep Med 2017; 13: 1029-1037.

35 Joosten SA, Leong P, Landry SA, et al. Loop gain predicts the response to upper airway surgery in patients with obstructive sleep apnea. Sleep 2017; 40: zsx094.

36 Salloum A, Rowley JA, Mateika JH, et al. Increased propensity for central apnea in patients with obstructive sleep apnea: effect of nasal continuous positive airway pressure. Am J Respir Crit Care Med 2010; 181: 189-193.

37 Bamagoos AA, Cistulli PA, Sutherland K, et al. Phenotyping using polysomnography to select obstructive sleep apnoea patients for mandible advancement device therapy. J Sleep Res 2017; 26: Suppl. 1, 5-33. 